\title{
The degree of mutual correlation of coordinate distributions of Mueller matrix elements biological tissues and diagnostics of their physiological state
}

\author{
Yuriy A. Ushenko ${ }^{1}$, Yuriy Ya. Tomka ${ }^{2}$, Alexander Dubolazov ${ }^{2}$ \\ ${ }^{1}$ Department of Correlation Optics, Chernivtsi National University, Chernivtsi 58012, Ukraine, \\ yuriyu@gmail.com \\ ${ }^{2}$ Optics and Spectroscopy Department, Chernivtsi National University, Chernivtsi 58012, Ukraine
}

\begin{abstract}
The paper is aimed at researching the effectiveness of differentiation of optical anisotropic property of organic crystal networks by means of the statistic and correlation analysis of new analytical parameter - the degree of mutual correlation of biological tissues Mueller matrices of different morphological structure and physiological state.
\end{abstract}

Keywords: polarization, correlation, biological tissue, statistics, Mueller matrix

\section{The main theoretical statements}

For actual biological tissues (BT) object fields including its images the change both of polarization and correlation characteristics is typical [1].

In the papers [2-6] the question bout defining the degree of mutual polarization between two points of coherent radiation field was examined. It was concluded that for general case of two elliptically polarized fluctuations

$$
E_{x_{1}}+E_{y_{1}} e^{-i \delta_{1}} \text { and } E_{x_{2}}+E_{y_{2}} e^{-i \delta_{2}}
$$

the degree of its polarization agreement is of the next analytical form

$$
|V|^{2}=\frac{\left(E_{x_{1}} E_{x_{2}}-E_{y_{1}} E_{y_{2}}\right)^{2}+4 E_{x_{1}} E_{y_{1}} E_{x_{2}} E_{y_{2}} e^{-i\left(\delta_{1}-\delta_{2}\right)}}{\left(E_{x_{1}}^{2}+E_{y_{1}}^{2}\right)\left(E_{x_{2}}^{2}+E_{y_{2}}^{2}\right)} .
$$

Ellis and Dogariu [7] called this parameter the degree of mutual polarization (DMP) of the points of field with coordinates $r_{1}$ and $r_{2}[8,9]$.

On the other hand, equation (2) is a "derivative" from the matrix equation solution

$$
\left(\begin{array}{l}
S_{1}^{*} \\
S_{2}^{*} \\
S_{3}^{*} \\
S_{4}^{*}
\end{array}\right)=\left\|\begin{array}{cccc}
1 & 0 & 0 & 0 \\
0 & f_{22} & f_{23} & f_{42} \\
0 & f_{32} & f_{33} & f_{43} \\
0 & f_{42} & f_{43} & f_{44}
\end{array}\right\| \cdot\left(\begin{array}{c}
S_{1}^{*} \\
S_{2}^{*} \\
S_{3}^{*} \\
S_{4}^{*}
\end{array}\right),
$$

where

$$
\begin{array}{ccc}
f_{22}=\cos ^{2} 2 \rho+\sin ^{2} 2 \rho \cdot \cos \delta ; & f_{23}=\cos 2 \rho \sin 2 \rho(1-\cos \delta) ; & f_{24}=\sin 2 \rho \sin \delta ; \\
f_{32}=\cos 2 \rho \sin 2 \rho(1-\cos \delta) ; & f_{33}=\sin ^{2} 2 \rho+\cos ^{2} 2 \rho \cos \delta ; & f_{34}=\cos 2 \rho \sin \delta ; \\
f_{42}=-\sin 2 \rho \sin \delta ; & f_{43}=-\cos 2 \rho \sin \delta ; & f_{44}=\cos \delta .
\end{array}
$$


$S_{i=1,2,3,4}^{0}$ and $S_{i=1,2,3,4}^{*}$ - Stokes vector parameters, which are uniquely concerned with complex amplitudes $\left(E_{x}, E_{y}\right)$ at the points with coordinates $r_{1}$ and $r_{2}$ through the known equations

$$
\begin{aligned}
& S_{1}=E_{x} E_{x}^{*}+E_{y} E_{y}^{*}, \\
& S_{2}=E_{x} E_{x}^{*}-E_{y} E_{y}^{*}, \\
& S_{3}=E_{x} E_{y}^{*}+E_{y} E_{x}^{*}, \\
& S_{4}=i\left(E_{x} E_{y}^{*}-E_{y} E_{x}^{*}\right) .
\end{aligned}
$$

On the basis of (3) and (5) one can obtain the analytical relation, which characterizes the correlation similarity of polarization manifestation of optical-anisotropic component in different points of biological tissue. Hereinafter such parameter will be called as the degree of mutual correlation (DMC) of biological tissue Mueller matrix $W$.

$$
W=\frac{1+A-B \cdot C}{2}
$$

where

$$
\begin{gathered}
A=\frac{f_{34}\left(r_{1}\right) f_{34}\left(r_{2}\right)}{\sqrt{\left(1-f_{44}^{2}\left(r_{1}\right)\right)\left(1-f_{44}^{2}\left(r_{2}\right)\right)}}, \\
B=\frac{f_{24}\left(r_{1}\right) f_{24}\left(r_{2}\right)}{\sqrt{\left(1-f_{44}^{2}\left(r_{1}\right)\right)\left(1-f_{44}^{2}\left(r_{2}\right)\right)}}, \\
C=f_{44}\left(r_{1}\right) f_{44}\left(r_{2}\right)+\sqrt{\left(1-f_{44}^{2}\left(r_{1}\right)\right)\left(1-f_{44}^{2}\left(r_{2}\right)\right)} .
\end{gathered}
$$

\section{The diagnostics of BT physiological state on the basis of statistical and correlation analysis of} the DMC distributions of their Mueller matrices

Two groups of BT samples were chosen as the objects of investigation:

1. Sound and degeneratively changed (osteoporosis) shin osseous tissue (OT);

2. Sound and dystrophic changed skeletal muscular tissue (MT).

The choice of such objects is conditioned by the following factors:

- optical mechanisms of changes of organic crystals structure of such tissues are different;

- for the osseous tissue the decrease of organic crystals birefringence (washing out of bone-salt crystals (hydroxyl apatite)) with permanent orientation of optical axis of birefringent collagenous fibrils occurs - "phase scenario";

- for MT disordering of optical axes directions of organic crystals (myosin fibrils) with permanent birefringence occurs - "orientation scenario".

Figures 1 and 2 present the DMC coordinate distributions of Mueller matrix of sound (fragment (a)) and degeneratively-dystrophic changed (fragments (b)) BT of both types.

Tables 1 and 2 present the set of statistic moments values, which characterize the DMC of OT (table 1) and MT (table 2) Mueller matrix. 


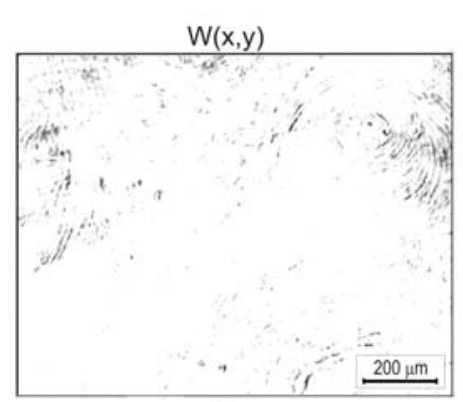

(a)

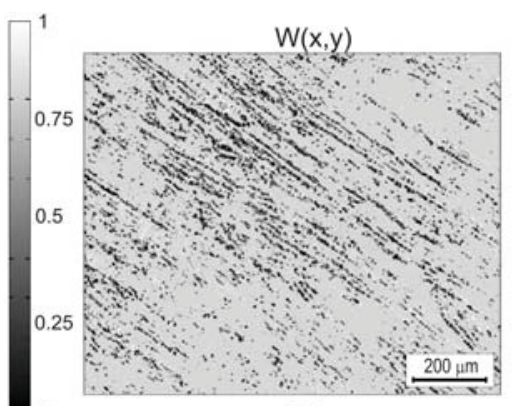

(b)

Figure 1. Two-dimensional distributions of the DMC $(W(x, y))$ of the sound (a) and degeneratively changed (b) OT Mueller matrix.

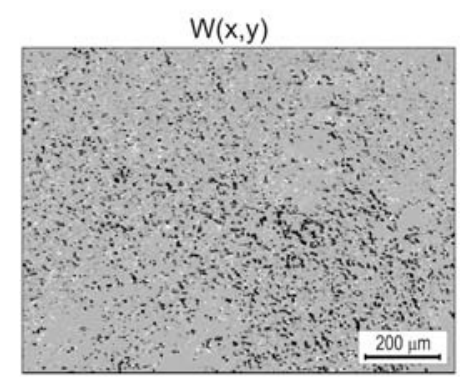

(a)
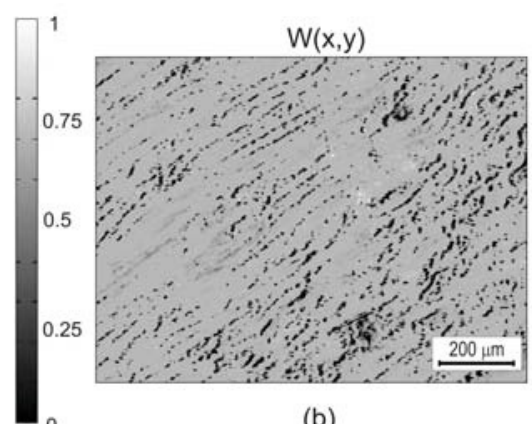

(b)

Figure 2. Two-dimensional distributions of the DMC $(W(x, y))$ of the sound (a) and degeneratively changed (b) MT Mueller matrix.

Table 1. Statistic moments of the 1st-4th orders of the DMC of OT (sound and degeneratively changed) Mueller matrix.

\begin{tabular}{|c|c|c|}
\hline Statistic moments & $\begin{array}{c}\text { OT } \\
\text { (sound, 21 samples) }\end{array}$ & $\begin{array}{c}\text { OT } \\
\text { (degeneratively changed, 18 samples) }\end{array}$ \\
\hline$M$ & $0.89 \pm 0.075$ & $0.41 \pm 0.036$ \\
\hline$\sigma$ & $0.023 \pm 0.0019$ & $0.093 \pm 0.0081$ \\
\hline$A$ & $8.35 \pm 0.68$ & $3.41 \pm 0.27$ \\
\hline$E$ & $21.5 \pm 2.07$ & $36.9 \pm 3.15$ \\
\hline
\end{tabular}

Table 2. Statistic moments of the 1st-4th orders of the DMC of MT (sound and dystrophic changed) Mueller matrix.

\begin{tabular}{|c|c|c|}
\hline Statistic moments & $\begin{array}{c}\text { MT } \\
\text { (sound, 19 } \\
\text { samples) }\end{array}$ & $\begin{array}{c}\text { MT } \\
\text { (dystrophic changed, 18 samples) }\end{array}$ \\
\hline$M$ & $0.62 \pm 0.057$ & $0.49 \pm 0.038$ \\
\hline$\sigma$ & $0.11 \pm 0.098$ & $0.14 \pm 0.012$ \\
\hline$A$ & $32.65 \pm 2.98$ & $21.14 \pm 1.83$ \\
\hline$E$ & $81.6 \pm 7.91$ & $43.8 \pm 3.72$ \\
\hline
\end{tabular}

Figure 3 and figure 4 illustrate the autocorrelation functions (ACF) of the parameter $W(x, y)$ of Mueller matrix of both types BT. 


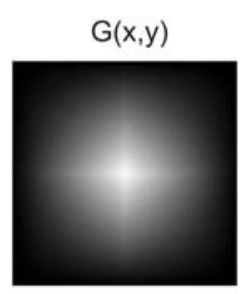

(a)

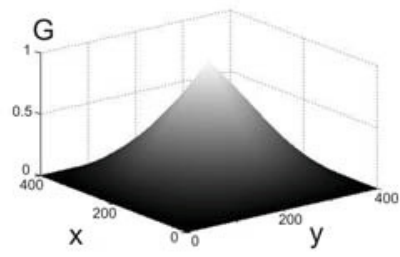

(c)

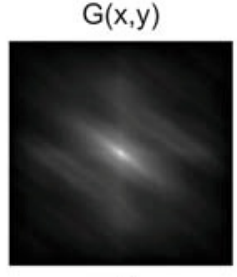

(b)

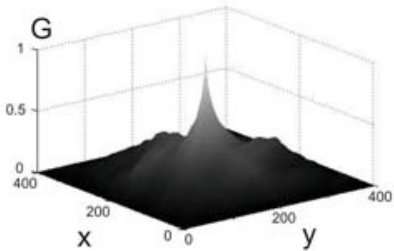

(d)

Figure 3. Two-dimensional autocorrelation functions $G(x, y)$ of parameter $W(x, y)$ of sound (a) and degeneratively changed (b) OT. Parts (c, d) correspond to three-dimensional representation of $G(x, y)$.

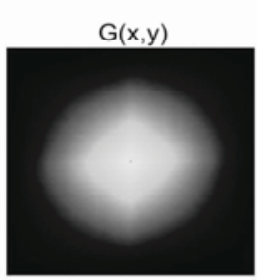

(a)

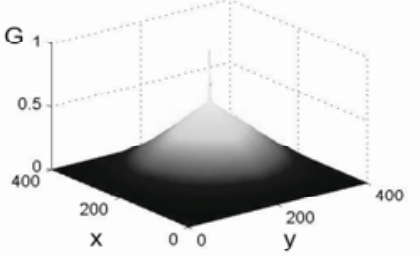

(c)
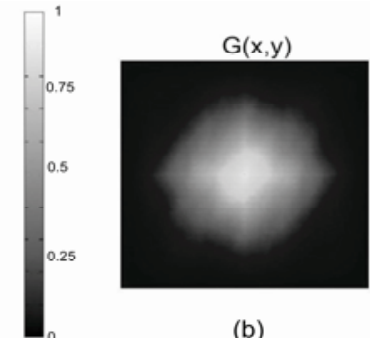

(b)

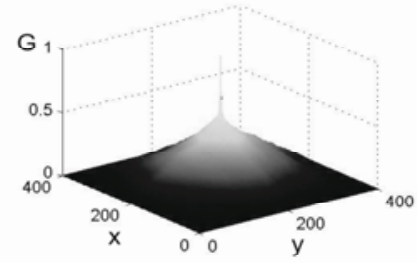

(d)

Figure 4. Two-dimensional autocorrelation functions $G(x, y)$ of parameter $W(x, y)$ of sound (a) and dystrophic changed (b) MT. Parts (c, d) correspond to three-dimensional representation of $G(x, y)$.

The statistic analysis of the DMC coordinate distribution $W(x, y)$ (tables 3 and 4 ) has detected the following:

- for sound and degeneratively changed OT the differences between the average and dispersion, the skewness and the kurtosis are of 1.5-4 times.

- for various conditions of MT the differences between statistic moments of the 1st-3rd orders are within of $30-50 \%$, the values of the kurtosis coefficient of the $W(x, y)$ distribution differs practically in 2-2.5 times.

The comparative analysis of correlation parameters has detected the essential distinctions in the values of half-width and dispersion of BT ACF oscillation (table 3). 
Table 3. Correlation parameters of DMC coordinate distribution of BT Mueller matrices.

\begin{tabular}{|c|c|c|c|c|c|}
\hline & \multicolumn{2}{|c|}{ OT } & \multicolumn{2}{c|}{ MT } \\
\cline { 2 - 3 } \cline { 5 - 6 } & sound & pathology & & sound & pathology \\
\hline $\begin{array}{c}L \cdot 10^{3}, \\
\mu m\end{array}$ & $0,6 \pm 0,04$ & $0,34 \pm 0,03$ & $\begin{array}{c}L \cdot 10^{3} \\
\mu m\end{array}$ & $0,25 \pm 0,02$ & $0,13 \pm 0,01$ \\
\hline$\Omega$ & $\approx 0$ & $0,01 \pm 0,001$ & $\Omega$ & $0,07 \pm 0,005$ & $0,005 \pm 0,0001$ \\
\hline
\end{tabular}

\section{Conclusions}

The degree of mutual correlation of Mueller matrix has been used for the first time for analysis of biological tissues optical properties. Such parameter characterizes the correlation similarity of polarization presentations of optical anisotropy of different areas organic crystals birefringent network.

It has been determined that physical reason of dispersion increasing and, vice versa, the skewness and the kurtosis decreasing of coordinate distribution of the degree of mutual correlation of biological tissue Mueller matrix caused by the increasing of distribution of organic crystal optical axes orientations because of dystrophic changes.

The inverse processes of statistic moments of the 2nd-4th orders value changes correspond to increasing of the phase shifts dispersion, which caused by dystrophic changed biological tissue organic crystals.

Thereupon the early (pre-clinical) discrimination between the optical properties of sound and degeneratively-dystrophic muscular and osseous tissues has been performed.

\section{References}

1. V. Sankaran, K. Schonenberger, J. T. Walsh, Jr., and D. J. Maitland, "Polarization discrimination of coherently propagation light in turbid media," Appl. Opt. 38, 4252-4261 (1999).

2. R. Hanau, "Interference of linearly polarized light with perpendicular polarizations," Am. J. Phys. 31, 303-304 (1963).

3. E. Collett, "Mathematical formulation of the interference laws of Fresnel and Arago," Am. J. Phys. 39, 1483-1495 (1971).

4. E. Collett, Polarized Light, Fundamentals and Applications (Marcel Dekker, New York, 1993).

5. C. Brosseau, Fundamentals of Polarized Light, A Statistical Optics Approach (Wiley, New York, 1998).

6. R. Barakat, "Analytic proofs of the Arago-Fresnel laws for the interference of polarized light," J. Opt. Soc. Am. A 10, 180-185 (1993).

7. E. Wolf, "Unified theory of coherence and polarization of random electromagnetic beams," Phys. Lett. A 312, 263-267 (2003).

8. E. Wolf, "Correlation-induced changes in the degree of polarization, the degree of coherence, and the spectrum of random electromagnetic beams on propagation," Opt. Lett.28, 1078-1080 (2003).

9. J.Ellis and A.Dogariu, "Complex degree of mutual polarization," Opt.Lett., 29, 536-538 (2004). 